\title{
Computer Use in Continued Fraction Expansions*
}

\author{
By Evelyn Frank
}

Abstract. In this study, the use of computers is demonstrated for the rapid expansion of a general regular continued fraction with rational elements for $\sqrt{ } C+L$, where $C$ and $L$ are rational numbers, $C$ positive. Formulas for the expansion are derived. Conditions for the periodicity are considered. A Fortran program for the algorithms is given, as well as sample continued fraction expansions. Up to the present, practically all studies have been concerned with continued fractions with partial numerators \pm 1 and partial denominators positive integers, due to difficulties in calculation. But now the use of computers makes possible the study of a much greater variety of continued fraction expansions.

1. Introduction. A general regular continued fraction has been defined [3] as a finite or infinite continued fraction

$$
b_{0}+\frac{a_{1}}{b_{1}}+\frac{a_{2}}{b_{2}}+\cdots, \quad a_{n+1}, b_{n} \neq 0, n=0,1, \cdots,
$$

with real numerical elements such that

$$
b_{n} \geqq 1+\left|a_{n+1}\right|, \quad\left|a_{n+1}\right| \geqq 1, n=0,1, \cdots,
$$

or with integral elements such that

$$
\left|a_{n}\right|=1, \quad b_{n} \geqq 1, \quad b_{n}+a_{n+1} \geqq 1, \quad n=1,2, \cdots .
$$

It has been shown in [3] that a continued fraction satisfying the latter conditions can always be transformed into one satisfying (1.2). A general regular continued fraction converges. The continued fraction expansion (1.1) for a real number $F_{0}$ is accomplished by a sequence of linear transformations

$$
F_{n}=b_{n}+\frac{a_{n+1}}{F_{n+1}}, \quad F_{n} \geqq 1, \quad n=0,1, \cdots .
$$

In [3] it was shown that, if

$$
\left|F_{n}-b_{n}\right|<1, \text { that is, } F_{n+1}>\left|a_{n+1}\right|, \quad n=0,1, \cdots,
$$

a general regular continued fraction expansion satisfying (1.2) converges to the generating number $F_{0}$. An expansion that satisfies (1.3) is semiregular (or regular if $a_{n+1}=1$ ) and always converges to the generating number (cf. [6]).

In [4] the author studied general regular continued fraction expansions with real rational numerical elements for $\sqrt{ } C+L$ with $C$ and $L$ rational numbers, $C$ positive. Conditions were also found for general regular expansions (1.1) for $\sqrt{ } C+L$ to be periodic. Simultaneously, the approximations $x_{n}$ to $\sqrt{ } C+L$ given by an extension of Newton's formula,

Received July 2, 1968, revised September 25, 1968.

* Presented to the American Mathematical Society. This research was sponsored in part by the National Science Foundation. 


$$
x_{n}=\frac{x_{i} \cdot x_{j}+C-L^{2}}{x_{i}+x_{j}-2 L},
$$

where $x_{i}$ and $x_{j}$ are certain previous approximations to the value $\sqrt{ } C+L$, were studied. Let $x_{i}=X_{i} / Y_{i}$ denote the $i$ th approximant of (1.1). In [4] a complete classification was given concerning which ones of the approximants $X_{i} / Y_{i}$ are also approximations to $\sqrt{ } C+L$ given by formula (1.6).

In this study, the use of computers is demonstrated for the rapid expansion of a general regular continued fraction with rational elements for $\sqrt{ } C+L$. The use of computers is especially convenient here since the same operations are repeated many times, and computation without computers is extremely arduous.

Furthermore, practically all studies up to this time have been concerned with the elements $a_{i}= \pm 1, b_{i}$ positive integers. But now the use of computers makes a study of continued fractions with rational elements no more difficult, since it takes almost no time to work out many examples.

In Section 2, formulas for the expansion of $\sqrt{ } C+L$ into a general regular continued fraction (1.1) with rational elements are derived. These are then modified for application to a computer program. In Section 3, conditions for the periodicity of these expansions are discussed. In Section 4 a summary of the calculation procedure is given, and sample problems computed for a particular class of general regular continued fraction expansions for $\sqrt{ } C+L$ (Table 1). These are given in order to show how easily general regular continued fraction expansions can be generated for a given binomial quadratic surd.

A Fortran program for the expansion of $\sqrt{ } C+L$ into a general regular continued fraction appears in the microfiche section of this issue of the journal.

\section{Mathematical Description.}

I. The first problem is the derivation of formulas for the computation of the expansion (1.1) for $\sqrt{ } C+L$. For a given binomial quadratic surd $\sqrt{ } C+L$, one writes

$$
\sqrt{ } C+L=\frac{\sqrt{ } D+P_{0}}{Q_{0}} .
$$

The continued fraction (1.1) is generated by a sequence of linear transformations

$$
F_{n}=b_{n}+\frac{a_{n+1}}{F_{n+1}}=\frac{\sqrt{ } D+P_{n}}{Q_{n}}, \quad n=0,1, \cdots .
$$

The elements must satisfy (1.2) or (1.3), and (1.5). The transformation (2.2) can be written

$$
\frac{\sqrt{ } D+P_{n}}{Q_{n}}=b_{n}+\frac{a_{n+1} Q_{n+1}}{\sqrt{ } D+P_{n+1}}, \quad n=0,1, \cdots .
$$

From this formula, the recurrence relations

$$
D-P_{n+1}^{2}=a_{n+1} Q_{n} Q_{n+1},
$$




$$
\begin{aligned}
P_{n+1} & =b_{n} Q_{n}-P_{n}, \\
Q_{n+1} & =\frac{b_{n}\left(P_{n}-P_{n+1}\right)+a_{n} Q_{n-1}}{a_{n+1}}=\frac{D-P_{n+1}^{2}}{a_{n+1} Q_{n}}, \quad n=0,1, \cdots, \\
Q_{-1} & =\frac{D-P_{0}^{2}}{Q_{0}} \quad\left(a_{0}=1\right),
\end{aligned}
$$

were derived in [4].

In the continued fractions considered, the $a_{n+1}, b_{n}, Q_{n-1}$, and $P_{n}, n=0,1, \cdots$, are rational numbers. Consequently, it is more convenient in the computation with computers for one to consider the continued fraction

$$
\frac{B_{0}}{D_{0}}+\frac{A_{1} / C_{1}}{B_{1} / D_{1}}+\frac{A_{2} / C_{2}}{B_{2} / D_{2}}+\cdots
$$

for $\sqrt{ } C+L=\left(\sqrt{ } D+P_{0} / R_{0}\right) /\left(Q_{0} / S_{0}\right)$. This is generated by the sequence of linear transformations

$$
\frac{\sqrt{ } D+P_{n} / R_{n}}{Q_{n} / S_{n}}=\frac{B_{n}}{D_{n}}+\frac{\left(A_{n+1} / C_{n+1}\right) \cdot\left(Q_{n+1} / S_{n+1}\right)}{\sqrt{ } D+P_{n+1} / R_{n+1}}, \quad n=0,1, \cdots .
$$

Thus, for the continued fraction (2.6), the recurrence relations (2.4) and (2.5) become

$$
\begin{aligned}
\frac{P_{n+1}}{R_{n+1}} & =\frac{B_{n} Q_{n} R_{n}-D_{n} P_{n} S_{n}}{D_{n} R_{n} S_{n}}, \\
\frac{Q_{n+1}}{S_{n+1}} & =\frac{\left[B_{n} C_{n} S_{n-1}\left(P_{n} R_{n+1}-P_{n+1} R_{n}\right)+A_{n} D_{n} Q_{n-1} R_{n} R_{n+1}\right] C_{n+1}}{A_{n+1} C_{n} D_{n} R_{n} R_{n+1} S_{n-1}} \\
& =\frac{C_{n+1} S_{n}\left(D R_{n+1}^{2}-P_{n+1}^{2}\right)}{A_{n+1} Q_{n} R_{n+1}^{2}}, \quad n=0,1, \cdots, \\
\frac{Q_{-1}}{S_{-1}} & =\frac{\left(D R_{0}{ }^{2}-P_{0}{ }^{2}\right) S_{0}}{Q_{0} R_{0}{ }^{2}} .
\end{aligned}
$$

Semiregular continued fractions (continued fractions that satisfy (1.3)) are not considered here, since semiregular expansions for $\sqrt{ } C+L$ have been treated by Perron [6], Goncalves [5], and many others. In fact, the author in [2] gave an Algol program for the expansion of $\sqrt{ } C+L$ into a regular continued fraction. With slight modifications, the expansion into a semiregular continued fraction could be similarly programmed. Consequently, only general regular continued fractions (2.6) that satisfy the conditions

$$
\begin{aligned}
\frac{B_{n}}{D_{n}} \geqq 1+\left|\frac{A_{n+1}}{C_{n+1}}\right|, \quad\left|\frac{A_{n+1}}{C_{n+1}}\right| \geqq 1, \quad C_{n+1}>0, \quad D_{n}>0, \\
\left|F_{n}-\frac{B_{n}}{D_{n}}\right|<1, \quad \text { i.e. } F_{n+1}>\left|\frac{A_{n+1}}{C_{n+1}}\right|, \quad n=0,1, \cdots,
\end{aligned}
$$

are treated here. As in the case of semiregular continued fractions, given are the positive integers $C_{n+1}$ and the positive or negative integers $A_{n+1}, n=0,1, \cdots$. Also given are the integers $P_{0}, Q_{0}$, and $D\left(R_{0}=S_{0}=1, A_{0}=C_{0}=1\right)$, and the generating 
number $F_{0}>1$. The positive integers $B_{n}$ and $D_{n}$ are computed from (2.9) with certain specific rules concerning their unique values. As discussed in [3], [4], the continued fraction (2.6) can always be transformed into one for which $\left|A_{n+1} / C_{n+1}\right|>1$, $B_{n} / D_{n}>1$, so it is henceforth assumed that these conditions hold.

If $\left(\sqrt{ } D+P_{0}\right) / Q_{0}$ is negative, one computes $(2.6)$ for $-\left(\sqrt{ } D+P_{0}\right) / Q_{0}$, and then multiplies $(2.6)$ by -1 . Furthermore, $\sqrt{ } D$ is taken as the positive root, since, if one is given $\left(-\sqrt{ } D+P_{0}\right) / Q_{0}$, one uses the equal value $\left(\sqrt{ } D-P_{0}\right) /\left(-Q_{0}\right)$.

If the expansion is periodic, one notes that one has obtained a complete period $p$ when the values of $P_{i} / R_{i}, Q_{i} / S_{i}$ are repeated. One must of course start with a periodic sequence (of period $r$ ) of the $A_{n+1} / C_{n+1}$. Then $p=r \cdot k$, where $k$ is a positive integer. (This is analogous to the case of semiregular continued fractions when one is given the $C_{n+1}=1$, and the $A_{n+1}$ are a given periodic sequence such that $\left|A_{n+1}\right|=1$.)

However, it may very well happen that there is a fore-period of $t$ terms, as in the expansion

$$
\begin{gathered}
\frac{G_{0}}{H_{0}}+\frac{M_{1} / N_{1}}{G_{1} / H_{1}}+\cdots+\frac{M_{t} / N_{t}}{G_{t} / H_{t}+K}=\frac{X_{t}+K \cdot X_{t-1}}{Y_{t}+K \cdot Y_{t-1}}, \\
K=\left(\frac{A_{1} / C_{1}}{B_{1} / D_{1}}+\cdots+\frac{A_{p} / C_{p}}{B_{p} / D_{p}}\right)+\left(\frac{A_{1} / C_{1}}{B_{1} / D_{1}}+\cdots+\frac{A_{p} / C_{p}}{B_{p} / D_{p}}\right)+\cdots,
\end{gathered}
$$

where the periodic part does not begin until the $(t+1)$ th term, and $X_{t}, Y_{t}$, denote the numerator and denominator, respectively, of the $t$ th approximant. This expansion is called mixed-periodic.

II. This portion of the mathematical description of the problem is concerned with additional formulas that one can use for further information about general regular expansions for $\sqrt{ } C+L$. These are analogous to the computations of the author in [2] on regular continued fraction expansions.

The recurrence relations for the $X_{n}$ and $Y_{n}$, the numerator and denominator, respectively, of the $n$th approximant of (2.6), are given by

$$
\begin{aligned}
X_{n}=B_{n} X_{n-1} / D_{n}+A_{n} X_{n-2} / C_{n}, \quad Y_{n} & =B_{n} Y_{n-1} / D_{n}+A_{n} Y_{n-2} / C_{n}, \\
n=1,2, \cdots, X_{-1} & =1, X_{0}=B_{0} / D_{0}, Y_{-1}=0, Y_{0}=1 .
\end{aligned}
$$

Also of interest is the application of formula (1.6) for the approximants of the expansions considered here. For that purpose it takes the form

$$
\begin{aligned}
x_{k p-s}= & \frac{Q_{0}{ }^{2} X_{k p-s}}{Q_{0}{ }^{2} Y_{k p-s}} \\
= & \frac{Q_{0}{ }^{2} X_{(k-r) p-s} X_{r p-1}+\left(D-P_{0}{ }^{2}\right) Y_{(k-r) p-s} Y_{r p-1}}{Q_{0}{ }^{2}\left(X_{(k-r) p-s} Y_{r p-1}+X_{r p-1} Y_{(k-r) p-s}\right)-2 P_{0} Q_{0} Y_{(k-r) p-s} Y_{r p-1}}, \\
\quad & \quad k=2,3, \cdots, r=1,2, \cdots, k-r \geqq 1, p=1,2, \cdots, s \leqq p .
\end{aligned}
$$

There are numerous other formulas of this type that could also be applied to the approximants of general regular continued fractions on a computer (cf. [1], [4]).

Finally, it is of great importance for one to know how good an approximation to the value of $\sqrt{ } C+L$ is an approximant $X_{n} / Y_{n}$. For that purpose, one can use the error formula (cf. [3]) 


$$
\frac{\left|\frac{A_{1} A_{2} \cdots A_{n}}{C_{1} C_{2} \cdots C_{n}}\right|}{Y_{n-1}\left(Y_{n}+Y_{n-1}\right)} \leqq\left|F_{0}-\frac{X_{n-1}^{*}}{Y_{n-1}^{*}}\right| \leqq \frac{\left|\frac{A_{1} A_{2} \cdots A_{n}}{C_{1} C_{2} \cdots C_{n}}\right|}{Y_{n-1}\left(Y_{n}-Y_{n-1}\right)} .
$$

Here $X_{n-1}^{*} / Y_{n-1}^{*}$ refers to the approximant $X_{n-1} / Y_{n-1}$ with all common factors removed from the numerator and denominator.

These three formulas have not been carried through in the Fortran program, since it has been the purpose of this paper to illustrate the usefulness of computers for expansions in general regular continued fractions, but the latter three formulas can likewise easily be applied to computers.

3. Conditions for the Periodicity of Expansion (2.6) for $\sqrt{ } C+L$. It is well known (cf. [5] or [6]) that an infinite semiregular continued fraction (1.3), into which a binomial quadratic surd can be expanded, is always periodic provided the partial numerators $a_{n+1}, n=0,1, \cdots$, are a given periodic sequence. Consequently, semiregular continued fractions are not considered here, and the discussion is concerned only with those general regular continued fractions satisfying conditions (1.2).

In [4] it was shown that a convergent infinite continued fraction that is periodic of period $p$ always represents a root of a quadratic equation provided the denominator of the $(p-1)$ th approximant of $(2.6)$ is not zero. Furthermore, it was shown that a general regular continued fraction (2.6) into which a binomial quadratic surd can be expanded, with the $A_{n+1}$ and $C_{n+1}$ a given periodic sequence, and with definite rules for the unique values of the $B_{n} / D_{n}$, is always periodic provided the $P_{n} / R_{n}$ in formulas (2.8) are integral.

That this is in general not the case can easily be seen if one writes down the successive values of $R_{n}$ and $S_{n}$ from formulas (2.8). It is seen that these values increase indefinitely, and it is only a fortunate cancellation of factors in the fractions $P_{n} / R_{n}$ and $Q_{n} / S_{n}$ that keeps the values of the numerators and denominators of these fractions bounded, with a consequent possibility for periodicity.

\section{Fortran Program and Sample Problems for a Particular Class of General} Regular Continued Fractions. In the microfiche section of this issue is given a Fortran program for a particular class of general regular continued fraction expansions for $\sqrt{ } C+L$. In this class, the periodic sequence $A_{n+1}=-1, C_{n+1}=1$ is chosen. Then the $B_{n} / D_{n}$ are calculated so that in the first formula in (2.8) the $P_{n+1} / R_{n+1}$ are integral, i.e. $R_{n+1}=1$. This is done in the following way: The denominators $D_{n}$ must be factors of $Q_{n}$. The factors $2,3, \cdots, Q_{n}$ are tried in that order as factors, and $D_{n}$ is set equal to the smallest one that is a factor. If none is a factor, then $D_{n}$ is set equal to 1 . The numerators $B_{n}$ are then chosen as multiples of $S_{n}$, and so chosen that $\left|F_{n}-B_{n} / D_{n}\right|<1$, and such that this difference is smallest, if there is a choice. Of course, since $A_{n+1}=-1, B_{n} / D_{n}>F_{n}$. Thus conditions (2.9) are satisfied.

It is clear that it is not always possible to find a $B_{n} / D_{n}$ that satisfies all these conditions. Below, in Table 1, is an illustration of a binomial quadratic surd that cannot be expanded into such a continued fraction. Of course, it would always be possible for one to alter, for example, the choice of $A_{n+1} / C_{n+1}$, so that a general regular continued fraction expansion is possible for a given binomial quadratic surd. 


\section{TABLE 1. Examples}

Example $1 \quad P=40 \quad Q=39 \quad D=46$

\begin{tabular}{rrrrrrrrrr}
\hline$N$ & \multicolumn{1}{c}{$A$} & $C$ & $B$ & $D$ & $P$ & $R$ & $Q$ & $S$ & $F N$ \\
0 & 1 & 1 & 4 & 3 & 40 & 1 & 39 & 1 & 1.19955 \\
1 & -1 & 1 & 117 & 14 & 12 & 1 & 98 & 39 & 7.47460 \\
2 & -1 & 1 & 28 & 15 & 9 & 1 & 195 & 14 & 1.13309 \\
3 & -1 & 1 & 65 & 42 & 17 & 1 & 1134 & 65 & 1.36318 \\
4 & -1 & 1 & 357 & 65 & 10 & 1 & 65 & 21 & 5.42198 \\
5 & -1 & 1 & 130 & 9 & 7 & 1 & 63 & 65 & 14.21986 \\
6 & -1 & 1 & 63 & 13 & 7 & 1 & 65 & 21 & 4.45275 \\
7 & -1 & 1 & 65 & 21 & 8 & 1 & 378 & 65 & 2.54193 \\
8 & -1 & 1 & 14 & 5 & 10 & 1 & 65 & 7 & 1.80733 \\
9 & -1 & 1 & 13 & 7 & 16 & 1 & 294 & 13 & 1.00738 \\
10 & -1 & 1 & 7 & 5 & 26 & 1 & 195 & 7 & 1.17680 \\
11 & -1 & 1 & 195 & 41 & 13 & 1 & 287 & 65 & 4.48031 \\
12 & -1 & 1 & 287 & 65 & 8 & 1 & 1170 & 287 & 3.62609 \\
13 & -1 & 1 & 65 & 41 & 10 & 1 & 861 & 65 & 1.26696 \\
14 & -1 & 1 & 1148 & 325 & 11 & 1 & 1625 & 287 & 3.14063 \\
15 & -1 & 1 & 975 & 287 & 9 & 1 & 2009 & 325 & 2.55314 \\
16 & -1 & 1 & 41 & 25 & 12 & 1 & 650 & 41 & 1.18473 \\
17 & -1 & 1 & 91 & 41 & 14 & 1 & 123 & 13 & 2.19650 \\
18 & -1 & 1 & 574 & 13 & 7 & 1 & 13 & 41 & 43.46733 \\
19 & -1 & 1 & 65 & 41 & 7 & 1 & 123 & 13 & 1.45667 \\
20 & -1 & 1 & 205 & 26 & 8 & 1 & 78 & 41 & 7.77020 \\
21 & -1 & 1 & 364 & 41 & 7 & 1 & 41 & 26 & 8.74001 \\
22 & -1 & 1 & 205 & 26 & 7 & 1 & 78 & 41 & 7.24456 \\
23 & -1 & 1 & 65 & 41 & 8 & 1 & 123 & 13 & 1.56236 \\
24 & -1 & 1 & 574 & 13 & 7 & 1 & 13 & 41 & 43.46733 \\
25 & -1 & 1 & 65 & 41 & 7 & 1 & 123 & 13 & 1.45667 \\
26 & -1 & 1 & 205 & 26 & 8 & 1 & 78 & 41 & 7.77020 \\
27 & -1 & 1 & 364 & 41 & 7 & 1 & 41 & 26 & 8.74001 \\
28 & -1 & 1 & 205 & 26 & 7 & 1 & 78 & 41 & 7.24456 \\
29 & -1 & 1 & 65 & 41 & 8 & 1 & 123 & 13 & 1.56236
\end{tabular}

Fore-period $=18 \quad$ Period $=6$

$\begin{array}{llll}\text { Example } 2 & P=9 & Q & \mathrm{D}=5\end{array}$

\begin{tabular}{rrrrrrrrrr}
\hline$N$ & $A$ & $C$ & $B$ & $D$ & $P$ & $R$ & $Q$ & $S$ & \multicolumn{1}{c}{$F N$} \\
0 & 1 & 1 & 13 & 5 & 9 & 1 & 5 & 1 & 2.52111 \\
1 & -1 & 1 & 40 & 3 & 4 & 1 & 3 & 5 & 12.67591 \\
2 & -1 & 1 & 8 & 5 & 4 & 1 & 5 & 1 & 1.52111 \\
3 & -1 & 1 & 40 & 3 & 4 & 1 & 3 & 5 & 12.67591 \\
4 & -1 & 1 & 8 & 5 & 4 & 1 & 5 & 1 & 1.52111 \\
& \multicolumn{8}{c}{ Period $=2$}
\end{tabular}

Example $3 \quad P=51 \quad Q=23 \quad D=73$

\begin{tabular}{cccccccccc}
\hline$N$ & $A$ & $C$ & $B$ & $D$ & $P$ & $R$ & $Q$ & $S$ & $F N$ \\
0 & 1 & 1 & 60 & 23 & 51 & 1 & 23 & 1 & 2.58887 \\
& $* * * N O$ & CHOICE & FOR & $B^{* * *}$ & & & & & \\
\hline
\end{tabular}

Furthermore, one could set down many different rules for the choice of the $A_{n+1} / C_{n+1}$ and of the consequent computation of the $B_{n} / D_{n}$. The type of expansion 
given here is described in detail in order to illustrate how a general regular expansion is generated. But it must be borne in mind that many other types of such expansions are possible.

The Fortran program was written for the IBM 360 which was the machine available for these computations. Floating-point arithmetic was used in order to obtain the maximum number of digits. Special care was also used in order to avoid round-off error.

A lowest terms subroutine (LTU) was used to reduce to lowest terms all $B_{n} / D_{n}$ and $Q_{n} / S_{n}$.

Department of Mathematics

University of Illinois at Chicago Circle

Chicago, Illinois 60680

1. E. Frank, "On continued fraction expansions for binomial quadratic surds," Numer. Math., v. 4, 1962, pp. 85-95. MR $25 \# 3603$.

2. E. Frank, "On continued fraction expansions for binomial quadratic surds. III," Numer. Math., v. 5, 1963, pp. 113-117. MR $27 \# 5355$.

3. E. Frank, "Continued fraction expansions with real numerical elements," Univ. Lisboa Revista Fac. Ci. A, (2), v. 12, 1968, pp. 25-40.

4. E. Frank, "Continued fraction expansions with rational elements for binomial quadratic surds," Univ. Lisboa Revista Fac. Ci. A, (2), 1969.

5. J. Vicente Gonçalves, "Sur le développement des irrationnalités quadratiques en fraction continue," Univ. Lisboa Revista Fac. Ci. A, (2), v. 4, 1955, pp. 273-282. MR 17, 18.

6. O. Perron, Die Lehre von den Kettenbriuchen, Band I: Elementare Kettenbriüche, Teubner, Verlagsgesellschaft, Stuttgart, 19.54. MR 16, 239. 